\title{
A Magnetically Navigated Microcannula for Subretinal Injections
}

\section{Journal Article}

\section{Author(s):}

Charreyron, Samuel (1); Boehler, Quentin (D); Nasrallah Danun, Aschraf (D); Mesot, Alexandre; Becker, Matthias; Nelson, Bradley (i)

Publication date:

2021-01

\section{Permanent link:}

https://doi.org/10.3929/ethz-b-000416852

\section{Rights / license:}

In Copyright - Non-Commercial Use Permitted

\section{Originally published in:}

IEEE Transactions on Biomedical Engineering 68(1), https://doi.org/10.1109/tbme.2020.2996013

\section{Funding acknowledgement:}

165564 - Soft Magnetic Robots: Modeling, Design and Control of Magnetically Guided Continuum Manipulators (SNF) 743217 - Soft Micro Robotics (EC) 


\title{
A Magnetically Navigated Microcannula for Subretinal Injections
}

\author{
Samuel L. Charreyron, Quentin Boehler, Aschraf N. Danun, Alexandre Mesot, Matthias Becker, \\ and Bradley J. Nelson
}

\begin{abstract}
Retinal disorders, including age-related macular degeneration, are leading causes of vision loss worldwide. New treatments, such as gene therapies and stem cell regeneration, require therapeutics to be introduced to the subretinal space due to poor diffusion to the active component of the retina. Subretinal injections are a difficult and risky surgical procedure and have been suggested as a candidate for robot-assisted surgery. We propose a different actuation paradigm to existing robotic approaches using remote magnetic navigation to control a flexible microcannula. A flexible cannula allows for high dexterity and considerable safety advantages over rigid tools, while maintaining the benefits of micrometer precision, hand tremor removal, and telemanipulation. The position of the cannula is tracked in realtime using near-infrared tip illumination, allowing for semiautomatic placement of the cannula and an intuitive user interface. Using this tool, we successfully performed several subretinal injections in ex-vivo porcine eyes under both microscope and optical coherence tomography visualization.
\end{abstract}

\section{INTRODUCTION}

D ISEASES of the retina are among the leading causes of vision loss and blindness worldwide [1]. Age-related macular degeneration (AMD), a disease which affects the central vision of older populations, is the leading cause of blindness in developed countries. AMD affects 200 million people worldwide, and its prevalence is projected to increase in developed countries to 288 million by 2040 due to an aging population [2].

AMD can be distinguished between a dry form that consists of $80 \%$ of cases and is currently untreatable, and a wet form which is associated with the formation of new blood vessels which may cause bleeding, retinal detachment, and a host of associated complications. Wet-AMD is treated by injecting antibodies acting on the vascular endothelial growth factor (VEGF) protein, which mediates the formation of vasculature. In standard practice, anti-VEGF drugs are injected into the vitreous humor, the clear viscoelastic body that fills the posterior cavity of the eye. While the technique is simple and is performed as an outpatient procedure in several countries, anti-VEGF injections must be performed on a regular basis and are associated with increased risks of cataract and elevated intraocular pressure [3]. Intravitreal injections of anti-VEGF

S.L. Charreyron, Q. Boehler, A. Mesot, B.J. Nelson are with the MultiScale Robotics Lab, ETH Zurich, Switzerland. A. Danun is now with the Product Development Group, ETH Zurich, Switzerland. M. Becker is with the Augenklinik, Triemlispital Zurich, Switzerland. (Correspondence email: samuelch@ethz.ch) Copyright (c) 2020 IEEE. Personal use of this material is permitted. However, permission to use this material for any other purposes must be obtained from the IEEE by sending an email to pubspermissions@ieee.org. drugs and corticosteroids are also used for treating some cases of diabetic retinopathy (DR) and diabetic macular endema (DME) [4]. Intravitreal injection allows for management of symptoms associated with these conditions, but it does not stop or reverse the degradation of the retina. A host of rarer inherited retinal diseases including retinitis pigmentosa (RP), Leber congenital amaurosis (LCA), Stargardt's disease, choroideremia, and retinoschisis, also lead to vision loss and total blindness in millions of patients worldwide. These diseases are, for the most part, untreatable [5].

There is active research in developing new therapeutics for treating retinal diseases, including gene therapies carried by viral vectors, stem cells, or artificial stimulation of the retina. Gene therapy using the adeno-associated virus (AAV) for mutations of the RPE65 gene that is associated with certain forms of inherited LCA, was successfully demonstrated in several clinical trials, resulting in the first-ever FDA-approved gene therapy. Treatments for other retinal disorders including choroideremia [6], and wet-AMD [7], are currently in clinical trials. Additionally, stem cell grafts of retina pigment epithelial (RPE) cells are being studied for treating AMD [8]. The promise of such therapies is that they could be used to stop or potentially reverse vision loss by correcting or re-engineering the genetic codes of retinal cells in the former, or stimulating regeneration of retinal cells in the latter. Therapies are being developed to bypass faulty photoreceptive pathways in the retina and also by artificial external stimulation in bionic eyes [9].

Many of these new retinal therapies are incompatible with intravitreal delivery. Therapeutics that are larger than smallmolecules show poor diffusion through the vitreous humor and across the inner limiting membrane (ILM), which separates the vitreous humor from the neuronal cells of the retina [5]. It has been suggested instead that drugs should be injected directly in the subretinal space (SRS), which separates the RPE cells from the photoreceptor cells of the retina. In subretinal drug-delivery, the neuronal retina is locally detached from the RPE by forming a subretinal bleb, into which therapeutics can later be introduced. The SRS exhibits several advantages for delivery. First, it has immune privilege, which is important to prevent carrier viruses or stem cells from being rejected by the patient's immune system. Only small-doses of potentially expensive therapeutics are required due to the small size of the SRS, and because injections can be targeted at specific parts of the retina. Subretinal injections (SRI) are reported to be safe when performed correctly, and subretinal blebs appear to resolve spontaneously after several days with little structural 
damage to the retina [10].

While SRI is heralded as the future pathway for retinal drug delivery, it is a challenging surgical procedure. The healthy neural retina has an average thickness of $212 \mu \mathrm{m}$ at the center of the fovea [11]. By comparison, hand-tremor during vitreoretinal surgery can reach as high as $100 \mu \mathrm{m}$ in amplitude [12]. There are complications associated with SRI including retinal tears, choroidal punctures, retinal holes, inflammation, and post-operative cataract formation [13]. While intraoperative optical coherence tomography (OCT) can provide surgeons with a high resolution cross-sectional view of the retina, and will probably be crucial to effectively monitor subretinal injections, OCT is not yet part of standard operating theater equipment [14]. While the cannula can be placed quickly, injections are performed slowly and can last several minutes due to the exceedingly small diameters of injection cannulas, and also to ensure safe detachment of the neural tissue [10]. Keeping a manual cannula stable during such long periods can be daunting, even for experienced surgeons. Additionally, there is no consensus on how many injections are required, or at which locations the injections should be performed. For certain diseases that affect the peripheral retina such as retinitis pigmentosa or choroideremia, a large number of injections may be required to sufficiently cover the entire targeted area [5].

Technological improvements have been suggested in view of the surgical difficulty of SRI. Automatic infusion systems can create slow and precise injections [15], [10], while subretinal bleb formation, foveal stretching, and existing macular holes can be monitored in real-time via intraoperative OCT [10], [13]. There has been prior work on developing robotic systems for assisting SRI. In [16], a teleoperated robot was developed and OCT-guided cannulation of bloodvessels was shown in ex-vivo porcine eyes. In-vivo SRI was shown in rabbit eyes using a comanipulated robot [17]. The first clinical study of robot-assisted SRI was reported in [18], where tissue plasminogen activator (tPA) was injected subretinally in order to treat subretinal hemorrhages in three patients. While there are some differences in the human-robot interface with some robots using a comanipulation and others a telemanipulation user interface, all existing approaches are variations on a common design of serially-actuated robots that manipulate conventional vitreoretinal tools using a remote center of motion (RCM) mechanism. Tools are made to rotate about the RCM at the sclerotomy, the small incision through which tools are introduced into the posterior eye, providing a large range-of-motion of the end-effector while minimizing mechanical stress at the incision. Nevertheless, robot-assisted devices have been shown to increase scleral forces when compared to manual surgery [19]. Other advantages which are common to all robotic approaches include hand-tremor removal, superhuman instrument tip placement precision, and hands-free stabilization of the tool inside the eye.

In this paper, we introduce a robotic system for performing SRI using a highly flexible injection cannula. The cannula can be navigated remotely, even in peripheral areas of the retina, using magnetic fields generated by an electromagnetic navigation system (eMNS). Magnetic steering is particularly interesting for vitreoretinal applications since it enables extremely precise actuation of devices with small diameters and high flexibility. Actuation is performed directly at the tip, and the cannula is mechanically constrained at the sclerotomies, which obviates the need for an RCM. Our approach shares many of the benefits of RCM vitreoretinal robots including tremor reduction, superhuman precision, and telemanipulation, but has the added benefits of increased safety and a large workspace, thanks to the extreme flexibility of the magnetically navigated cannula. This is of particular interest for SRI, which is a technically difficult surgical procedure and is associated with a number of risks including retinal tears, macular holes, or retinal detachments.

The contributions of this paper are the following. 1) The design of a magnetically-navigated injection cannula and insertion unit. 2) The implementation of a robust and noninvasive approach for precise tracking of the cannula using an embedded optical fiber providing near-infrared (NIR) illumination of the cannula tip. 3) The introduction of a simple kinematic model for microcannula motion. 4) Two modes of operation. A teleoperated mode, and semi-automated placement of the cannula tip using real-time image-based tracking through a standard surgical microscope providing an intuitive humanrobot interface. 5) The demonstration of subretinal injections in ex-vivo porcine eyes under both microscope and OCT guidance.

\section{MATERIALS}

\section{A. Magnetic Microcannula}

The microcannula design is shown in Fig. 1. It consists of a polyolefin elastomer (POE) tubular shaft (Dow Engage 8540, manufactured by Biogeneral Inc., San Diego, CA) with an outer diameter (OD) of $250 \mu \mathrm{m}$ and an inner-diameter (ID) of $190 \mu \mathrm{m}$ and length of $10 \mathrm{~cm}$. A custom axially-magnetized $\mathrm{Nd}$ FeB tubular permanent magnet (CK Magnet, Nanjing, China), with $400 \mu \mathrm{m}$ OD, $270 \mu \mathrm{m}$ ID, and $2 \mathrm{~mm}$ length, is attached to the flexible shaft at its distal end. A glass capillary with $80 \mu \mathrm{m}$ OD, $50 \mu \mathrm{m}$ ID (VitroCom, Mountain Lakes, NJ) is inserted inside the lumen of the shaft and protrudes the magnet by $1 \mathrm{~mm}$. The 44 gauge $(\mathrm{G})$ capillary is the most distal component of the cannula through which fluid flows and also creates a retinomy through which fluid enters the SRS. An optical fiber with $70 \mu \mathrm{m}$ OD (Doric Lenses, Franquet, QC) runs through the lumen of the cannula and features a tip diffusing section that scatters light over a wide angle. The diameter of the cannula is maximal at the magnetic section and is a $27 \mathrm{G}$ instrument. The cannula is connected via a silicone tube to a custom designed Y-connector. The Y-connector connects the cannula to a $10 \mathrm{~mW} 785 \mathrm{~nm}$ NIR laser (LPS-785-FC, Thorlabs Inc., Newton, $\mathrm{NJ}$ ) via a FC/PC connection, and to a syringe via a Luer connection.

\section{B. Mechanical Insertion Unit}

The microcannula is inserted and retracted through a sclerotomy via a mechanical insertion unit (MIU) shown in Fig. 2 d). The MIU consists of a piezoelectric linear positioner (SLC-2490, SmarAct GmbH, Oldenburg, Germany), which 


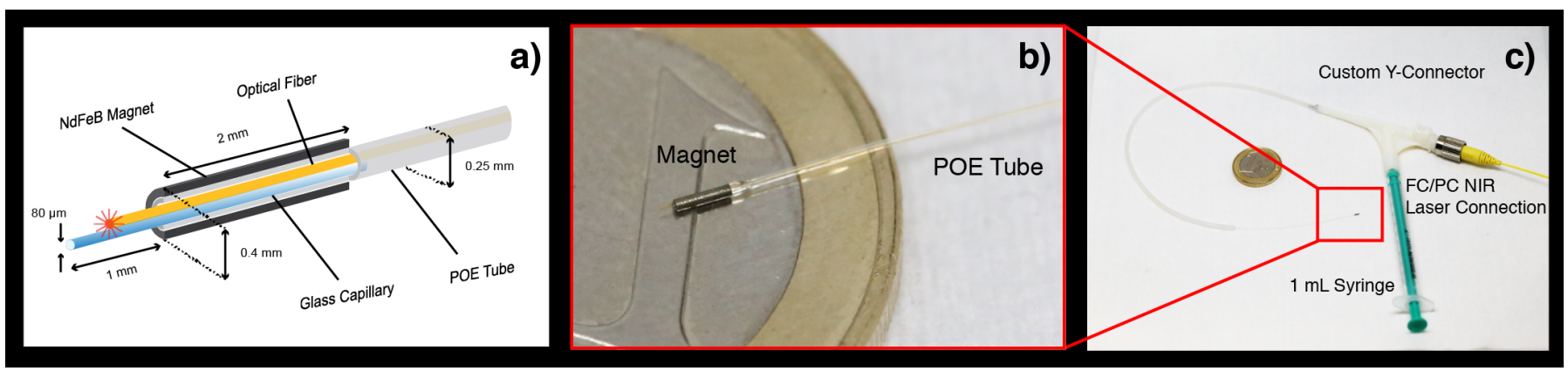

Fig. 1. Magnetic subretinal injection microcannula. a) Diagram showing the components of the distal end of the cannula. b) Close-up view of the distal end of the cannula. 1 euro coin shown for scale. c) Injection system. The proximal end of the cannula is connected to a custom-made Y-connector. The Y-connector provides a Luer connection to a $1 \mathrm{~mL}$ injection syringe, and a FC/PC connection to a NIR laser.

drives the insertion of the cannula and an introducer system, consisting of three telescoping non-magnetic stainless steel tubes which enter a $20 \mathrm{G}$ vitrectomy trocar. The introducer system was designed to guide the very flexible shaft of the cannula and to bypass the valve of the vitrectomy trocar. The MIU is mounted on an articulated arm to facilitate placement of the unit with respect to the eye. The insertion of the cannula is always controlled by a human operator. The operator can best assess how far the catheter must be inserted to contact the retina by observing visual cues such as shadows cast by the tool on the retina, buckling of the flexible shaft, or by using an imaging modality providing depth information such as OCT.

\section{Optical Tracking}

The posterior eye is visualized through the pupil using a microscope (M80, Leica, Wetzlar, Germany). A wideangle viewing system (BIOM, Oculus, Wetzlar, Germany) is mounted on the microscope and allows for visualization of a large part of the fundus. For the semi-automated mode of control, the microcannula's tip position was tracked automatically in the microscope fundus image. In vitreoretinal surgery, illumination is often provided by a handheld light pipe connected to an external light source, which creates a directional light source and thus inhomogeneous luminosity over the entire eye. Illumination and image quality depends on the opacity of the lens and cornea. To obtain tracking robust to varying illumination and visibility conditions, an active tracking system consisting of NIR illumination at the tip of the microcannula, and image processing to detect the tip position in real-time was implemented. NIR illumination has several advantages over visible light. First, NIR light is scattered less by the cornea and crystalline lens than visible light. Enhanced sensitivity of the tracking system without interference with the surgeon's view of the fundus could be obtained by separating the tip illumination from the visible spectrum. Additionally, since the NIR tip light source intensity is considerably lower than that of the light-pipe used to illuminate the entire cavity, this has the added benefit of minimizing intrusiveness to the patient.

The tracking system comprised two cameras connected to the camera port of the microscope via a beamsplitting mirror. A shortpass filter with a cutoff wavelength of $700 \mathrm{~nm}$ was placed in front of a color camera (acA2440-35uc Basler AG, Ahrensburg, Germany) which imaged the fundus. A bandpass filter with a passband spectrum of $780 \pm 10 \mathrm{~nm}$ was placed in front of a monochrome camera (acA2040-90um Basler AG), which imaged only the light emitted by the tracking laser, appearing as a bright spot on the otherwise dark background. This can be seen in Fig. 2 e). The NIR image was processed in real-time using image thresholding and contour detection from the OpenCV library [20] to detect the center of the laser spot, which was registered to the visible image using the calibrated homography.

\section{Remote Magnetic Navigation}

All experiments were performed in the OctoMag [21], an eight-electromagnet eMNS shown in Fig. 2 c). The magnetic fields generated inside the workspace depend on the electrical current running through the electromagnets of the eMNS. The eye was placed in the center of the workspace. The maximum magnetic field intensity was set to $40 \mathrm{mT}$, the maximum operating intensity of the OctoMag. For simplicity, it was assumed that the magnetic field distribution was uniform over the entire worskpace and coincided with the field at the center of the electromagnets. It was numerically determined that in the worst case, the magnetic field at a distance of $1.3 \mathrm{~cm}$, corresponding to the anatomical radius of a human eye, would deviate at most $26 \%$ from its value at the center of the workspace. The maximum forces that can applied by an eMNS using magnetic gradients are on the order of gravity [22, p. 62], which does not affect the cannula's deflection as significantly as magnetic torque. Therefore, the angular error of the magnetic field is the main limitation for accuracy, and this was determined to be $11.5^{\circ}$ in the worst case. For a tool of length $L=21 \mathrm{~mm}$, this results in a worst case displacement of $4.2 \mathrm{~mm}$. Inhomogeneous magnetic fields can nevertheless be compensated by closing the loop either by the human operator or using feedback control. Videos showing the cannula and system can be seen in supplementary video 1 .

\section{MODELS}

\section{A. Microcannula Kinematics Model}

The magnetic microcannula was modeled as a magnetic continuum robot. The distal end consists of the magnet and 


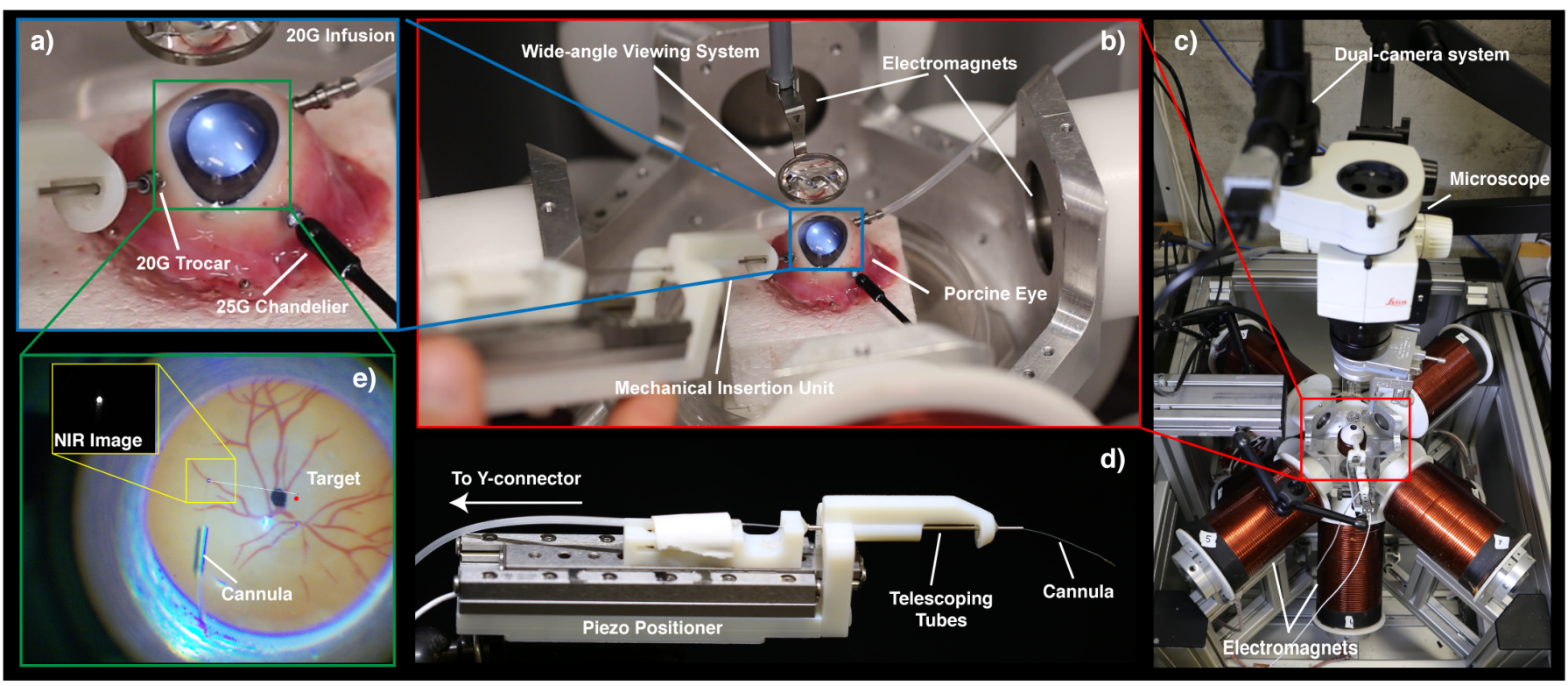

Fig. 2. The subretinal injection system. a-b) A porcine eye is placed in the center of the OctoMag eMNS. The eye is connected to a pars plana vitrectomy system with chandelier illumination and saline infusion. The fundus is viewed through a non-contact wide-angle viewing system. The MIU is docked to $20 \mathrm{G}$ trocar and inserts the cannula into the eye through the pars plana. c) Wide view of the OctoMag, microscope, and tracking system. d) The MIU. A piezo-actuaded positioner inserts the cannula through a series of 3 telescoping tubes. e) The fundus view with the cannula tip tracked in the NIR image.

a)

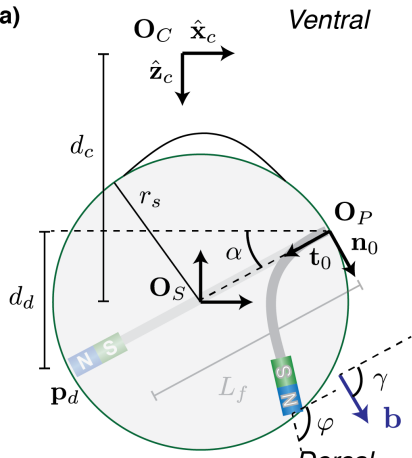

Dorsal b)

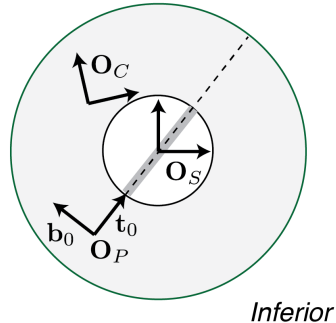

Fig. 3. Diagram of the kinematic model of the cannula. The spherical model of the eye is represented by the green circles. The arrows represent coordinate frames of the camera, sphere, and proximal point at the sclerotomy. a) View in the sagittal plane b) View in the frontal plane.

capillary end-effector, while the proximal end consists of the fixed sclerotomy. The kinematics of the cannula are governed by two control inputs: a) the length, which is controlled by inserting and retracting the cannula from the sclerotomy b) the magnetic fields generated by the eMNS. The permanent magnet at the tip of the cannula experiences magnetic torques which tend to align it with the magnetic field generated by the eMNS, causing the cannula to bend in the direction of the applied field. Under the assumption of a uniform and isotropic one-dimensional rod, which is only under the effects of the bending moment induced by the magnetic torque on the distal magnet, the flexible part of the cannula can be represented as an arc with a constant curvature [23]. The positions along the length of the cannula are constrained to a manipulation plane, which is spanned by $\mathbf{t}_{\mathbf{0}}$ and $\mathbf{b}$, the magnetic field vector at the

position of the distal magnet, provided that $\mathbf{b}$ is not parallel to $\mathbf{t}_{0}$. Adjusting $\mathbf{b}$ and the cannula length results in $3 \mathrm{D}$ control of the cannula tip. The equilibrium results from the calculus of variations on the balance of potential energies, and is exact provided the aforementioned assumptions. In practice however, the motion of the rod deviates from that of the idealized model, and feedback control is therefore used for position control. The kinematic model of the cannula is displayed in Fig. 3.

The point exiting the sclerotomy trocar is designated as the proximal point $\mathbf{O}_{p} \in \mathbb{R}^{3}$. For a positive length $l \in\left[0, L_{f}\right]$ where $L_{f}$ is the length of the flexible part of the cannula, the position $\mathbf{p}(l) \in \mathbb{R}^{3}$ can be written as

$$
\mathbf{p}(l)=\frac{1}{k}\left[(1-\cos \varphi) \hat{\mathbf{t}}_{0}+\sin \varphi \hat{\mathbf{n}}_{0}\right]+\mathbf{O}_{p}
$$

while the tangent vector $\mathbf{t}(l) \in \mathbb{R}^{3}$ to the arc at $l$ is

$$
\mathbf{t}(l)=\sin \varphi \hat{\mathbf{t}}_{0}+\cos \varphi \hat{\mathbf{n}}_{0}
$$

$\hat{\mathbf{t}}_{0} \in \mathbb{R}^{3}$ and $\hat{\mathbf{n}}_{0} \in \mathbb{R}^{3}$ are the tangent and normal vectors respectively to the arc at the proximal point, and along with $\hat{\mathbf{b}}_{0} \in \mathbb{R}^{3}$ form an orthonormal basis for the manipulation plane. $k \in \mathbb{R}^{+}$is the strictly positive curvature of the arc, while $\varphi=k l$ is the angle between $\hat{\mathbf{t}}_{0}$ and $\mathbf{t}(l)$. The curvature can be determined by solving the following equilibrium equation, derived in [23].

$$
k E_{f} I_{f}-M B_{0} \sin (\gamma-\varphi)=0
$$

where $E_{f}$ is the Young's modulus and $I_{f}$ the second moment of area of the flexible segment $M$ is the magnetization of the distal magnet and $B_{0}$ is the magnetic field intensity. $\gamma$ is the angle between the magnetic field vector at the magnet position and $\hat{\mathbf{t}}_{0}$. 
For the case where the cannula is straight and $k=0$, the equations become

$$
\begin{aligned}
\mathbf{p}(l) & =l \hat{\mathbf{t}}_{0} \\
\mathbf{t}(l) & =\hat{\mathbf{t}}_{0}
\end{aligned}
$$

For lengths $l>L_{f}$ where $L_{f}$ is the length of the flexible segment and $L$ is the total length of the cannula, the position becomes

$$
\begin{aligned}
\mathbf{p}(l) & =\mathbf{p}\left(L_{f}\right)+\left(l-L_{f}\right) \mathbf{t}\left(L_{f}\right) \\
\mathbf{t}(l) & =\mathbf{t}\left(L_{f}\right)
\end{aligned}
$$

\section{B. Optics Model}

The fundus image seen through the microscope optics is modeled as if it was generated by a pinhole camera. For simplicity, distortion caused by the optical system formed by the crystalline lens of the eye, the wide-angle viewing system, microscope optics, and the lenses on the dual-camera system was ignored. A point $\mathbf{p} \in \mathbb{R}^{3}$ is projected on the image as the $2 \mathrm{D}$ point $\mathbf{p}^{i} \in \mathbb{R}^{2}$.

$$
\mathbf{p}^{i}=\operatorname{proj}_{C}(\mathbf{p})
$$

The projection function is defined as follows where $\tilde{\mathbf{p}}^{i} \in \mathbb{R}^{3}$ is the image point in homogeneous coordinates corresponding to $3 \mathrm{D}$ point $\tilde{\mathbf{p}} \in \mathbb{R}^{4}$, also in homogeneous coordinates.

$$
\tilde{\mathbf{p}}^{i}=\underbrace{\left[\begin{array}{ccc}
f & 0 & w / 2 \\
0 & f & h / 2 \\
0 & 0 & 1
\end{array}\right]}_{\mathbf{K}_{c}} \underbrace{\left[\begin{array}{cc}
\mathbf{R}_{c} & \mathbf{t}_{\mathbf{c}} \\
0 & 1
\end{array}\right]}_{\mathbf{T}_{c}} \tilde{\mathbf{p}}
$$

where $w$ and $h$ are the width and height of the sensor in pixels, $\mathbf{O}_{c}^{i}=\left[\begin{array}{ll}w / 2 & h / 2\end{array}\right]^{T}$ is the principal point assumed to be at the center of the image, and $f$ is the camera focal length in pixels. The focal length is $120 \mathrm{~mm}$ was taken from the specifications of the microscope. These form the intrinsic parameters of the camera model, grouped into matrix $\mathbf{K}_{c}$. The extrinsic parameters of the camera model are a transformation matrix $\mathbf{T}_{c} \in \mathrm{SE}(3)$ transforming $3 \mathrm{D}$ points from a reference frame to a frame centered on the camera center $\mathbf{O}_{c}$.

\section{C. eMNS Model}

The eMNS is modeled mathematically as a number of magnetic multipoles, of which the magnetization depends on the currents running through the electromagnets [24]. The magnetic field at a given position is determined by the following linear equation

$$
\mathbf{b}(\mathbf{p})=\mathbf{A}(\mathbf{p}) \mathbf{i}
$$

where $\mathbf{b} \in \mathbb{R}^{3}$ is the magnetic flux density at position $\mathbf{p} \in \mathbb{R}^{3}$ in the workspace of the eMNS, $\mathbf{i} \in \mathbb{R}^{8}$ is a vector of currents running through the eight electromagnets of the eMNS, and A $\in \mathbb{R}^{3 \times 8}$ is the actuation matrix from the multipole model.

\section{Semi-automated Control}

The teleoperation mode consists in the human operator modifying both the magnetic field and the insertion length of the cannula in order to navigate to a desired location. In contrast, in the semi-automated mode, first introduced in [25], the magnetic field is modified automatically by observing the difference between the cannula's tip position in the image, and a desired position in the image. The control task is shared nonetheless with the human operator who both manipulates the desired image position, and controls the insertion of the cannula via the MIU.

Position control of the cannula tip is performed similar to [26]. Nevertheless, a shared-control strategy was employed as in [25], where the insertion of the cannula was performed by a human operator, while the magnetic positioning of the distal tip could be performed by an automatic controller.

The control inputs are $\mathbf{u}=\dot{\mathbf{b}}^{i} \in \mathbb{R}^{2}$, the time-derivatives of $\mathbf{b}^{i}$, the magnetic field in the image plane. We chose to control the magnetic field in the image plane and not the $3 \mathrm{D}$ magnetic field or the rotation of a 3D magnetic field with a fixed intensity, in order to avoid multiple catheter configurations which have the same projected distal point in the image. For example, a magnetic field pointing into the image and out of the image may result in catheter configurations where the distal positions appear at the same point in the image. See points $\mathbf{p}_{a}$ and $\mathbf{p}_{b}$ on Fig. $4 \mathrm{a}$ ). Controlling the magnitude of the fields in the image plane avoids such situations, while covering a workspace that contains almost the entire posterior hemisphere of the eye.

The control state is $\mathbf{x}=\left[\begin{array}{ll}\mathbf{p}_{d} & \mathbf{b}^{i}\end{array}\right]^{T} \in \mathbb{R}^{5}$ where $\mathbf{p}_{d}$ is the $3 \mathrm{D}$ distal position of the cannula from (6). Assume that $L$, the insertion length of the cannula, which is itself controlled by a human operator, is known. Also assume that the microcannula's dynamics are much faster than $\mathbf{u}$. The time derivative of the state is expressed as the following.

$$
\begin{aligned}
\dot{\mathbf{x}} & =g(\mathbf{x}) \mathbf{u} \\
g(\mathbf{x}) & =\left[\begin{array}{c}
\frac{\partial \mathbf{p}_{d}}{\partial \mathbf{b}^{i}} \\
\mathbf{I}_{2 \times 2}
\end{array}\right]
\end{aligned}
$$

The image projection of $\mathbf{p}_{d}$ is tracked, and the observation function is thus

$$
\mathbf{y}=h(\mathbf{x})=\operatorname{proj}_{C}\left(\mathbf{p}_{d}\right)
$$

The time derivative of the observation is

$$
\dot{\mathbf{y}}=\frac{\partial h(\mathbf{x})}{\partial \mathbf{x}} \dot{\mathbf{x}}=\underbrace{\frac{\partial h(\mathbf{x})}{\partial \mathbf{x}} g(\mathbf{x})}_{\mathbf{J}(\mathbf{x})} \mathbf{u}
$$

The Jacobian matrix $\mathbf{J}(\mathbf{x}) \in \mathbb{R}^{2 \times 2}$ relates changes in the magnetic field to changes in the image projection of the distal position. The control error $\mathbf{e} \in \mathbb{R}^{2}$ is the difference between a target position $\mathbf{p}_{t}^{i}$ in the image, and the tip position observed in the same image.

$$
\mathbf{e}=\mathbf{y}-\mathbf{p}_{t}^{i}
$$


Provided that $\mathbf{p}_{d}$ is within the cannula's workspace, $\mathbf{J}$ is of full rank, and using the following proportional control law results in $\mathbf{e} \rightarrow \mathbf{0}$ exponentially as $t \rightarrow \infty$.

$$
\mathbf{u}=-\mathbf{J}^{\dagger}(\mathbf{x}) \mathbf{K}_{p} \mathbf{e}
$$

where $\mathbf{K}_{p} \in \mathbb{R}^{2}$ is a matrix of proportional gains, and $\mathbf{J}^{\dagger}$ is the Moore-Penrose inverse of $\mathbf{J}$.

In addition to controlling the magnetic field in the image plane, a magnetic field offset points downwards into the image similarly to [27], depending on the distance between the distal position predicted by the cannula model and the target position projected onto the eye sphere $\mathbf{p}_{t} \in \mathbb{R}^{3}$.

$$
\mathbf{b}^{\prime}=\left[\begin{array}{c}
\mathbf{b}^{i} \\
k_{z}\left\|\mathbf{p}_{d}-\mathbf{p}_{t}\right\|
\end{array}\right]
$$

where $k_{z} \in \mathbb{R}$ is a scalar proportional gain. The magnetic field is scaled such that it never exceeded $b_{\max }=40 \mathrm{mT}$, the maximum field rating of the OctoMag.

$$
\mathbf{b}= \begin{cases}\mathbf{b}^{\prime} & \text { if }\left\|\mathbf{b}^{\prime}\right\|<b_{\max } \\ \mathbf{b}^{\prime} / b_{\max } & \text { otherwise }\end{cases}
$$

The gain parameters $\mathbf{K}_{p}$ and $k_{z}$ were determined experimentally.

\section{Model Calibration}

\section{A. Calibration of the Two-camera System}

The images of the NIR camera and the fundus camera are related by a homography. A point in the NIR image $\tilde{\mathbf{p}}_{n} \in \mathbb{R}^{3}$ expressed in homogeneous coordinates is related to a point $\tilde{\mathbf{p}}_{r} \in \mathbb{R}^{3}$ in the color fundus image by the homography matrix $\mathbf{H}_{r, n}$

$$
\tilde{\mathbf{p}}_{r}=\mathbf{H}_{r, n} \tilde{\mathbf{p}}_{n}
$$

$\mathbf{H}_{r, n}$ was computed by detecting the corners of a chessboard of known shape in both camera views, and using the direct linear transform (DLT) algorithm [28]. Because the NIR and fundus camera were fixed between experiments, $\mathbf{H}_{r, n}$ did not need to be recomputed unless the cameras were dissassembled from the camera port.

\section{B. Static Model Parameters}

A number of model parameters could be determined either by specifications from the manufacturers or by measurements. The eye was modeled as a sphere of radius $r_{s}=13 \mathrm{~mm}$ based on anatomical averages of human [29] and porcine [30] eyeballs. Although the flexible segment comprised a tube and optical fiber, it is modeled as as a hollow tube of a uniform material. By measuring force, and deflections of the flexible segment, and using Euler-Bernoulli beam theory, the Young's modulus of the segment was estimated to be $156.9 \mathrm{MPa}$. The distal permanent magnet's magnetization was measured with a vibrating scanning magnetometer to be $0.15 \mathrm{~A} \mathrm{~m}^{2}$. The remaining parameters were determined from specifications from the manufacturers.

\section{Pre-experiment Calibration}

The surgical microscope is often moved and adjusted during vitreoretinal surgery depending on the needs of the surgeon, patient specifics, or in order to visualize different parts of the posterior eye. The sclerotomies for inserting the vitreoretinal tools including the cannula were made manually using standard vitreoretinal trocar blades. For that reason, we developed a simplified calibration procedure to estimate the relative poses of the camera, trocars, and eyeball.

The distance of the camera to the sphere center $d_{c}$ is much larger than $d_{d}$, the vertical distance between the distal and proximal positions. Therefore, lines along the cannula axis were scaled by a constant factor $s_{c}=\frac{f}{d_{c}}$. Measuring a line of known length in the image determined $d_{c}$. The camera's optical axis $\hat{\mathbf{z}}_{c}$ was assumed to be parallel to the pupillary (dorsal - ventral) axis of the eye.

When no magnetic field was applied, the cannula was assumed to follow a line starting at its proximal point $\mathbf{O}_{p}$ and along the proximal tangent line $\hat{\mathbf{t}}_{0}$. $\hat{\mathbf{t}}_{0}$ was assumed to be inclined by a known angle $\alpha=30^{\circ}$ into the image plane, which was consistent with standard vitrectomy practice [31]. The length of the cannula $L$ was known at all times since it was set by the MIU.

Once the straight cannula was inserted through the sclerotomy and sufficiently visible by the camera, a line was traced following the axis of the cannula on the image represented by $\hat{\mathbf{t}}_{0}^{i}$. Combined with the previous assumptions, this determined $\hat{\mathbf{t}}_{0}$ with respect to the camera frame. Knowing the insertion length $L$ of the cannula, and the distal position in the image $\mathbf{p}_{d}^{i}$ determined the proximal point or sclerotomy position using the following formula

$$
\mathbf{t}_{p, c}=\left[\begin{array}{c}
\frac{\mathbf{p}_{d}^{i}-\mathbf{O}_{c}^{i}}{s_{c}}+L \hat{\mathbf{t}}_{0}^{i} \\
d_{c}
\end{array}\right]
$$

The cannula was assumed to be pointing towards the sphere center and knowing the sphere radius $r_{s}$ determined $\mathbf{t}_{s, c}$, the translation between the camera and sphere frames.

$$
\mathbf{t}_{s, c}=r_{s} \hat{\mathbf{t}}_{0}+\mathbf{t}_{p, c}
$$

Finally, we accounted for some in-plane rotation between the coordinate frames of the camera and of the eMNS, since the microscope was mounted on a swivelable articulated arm. The rotation was estimated by applying a magnetic field along the axis $\hat{\mathbf{t}}_{0}$ assuming that the camera frame was aligned with the MNS frame, which caused the cannula to bend slightly due to angular mismatch between the two frames. By measuring the distal tangent axis in the image, the rotational error between frames was measured. The center of the sphere and of the eMNS workspace were assumed to coincide, and without loss of generality, the sphere and eMNS share the same coordinate frame. The coordinate frames of the camera, proximal point, and sphere are shown in Fig. 3. We developed a graphical software tool for performing the calibration, and a screen-capture of the calibration process can be seen in supplementary video 2 . 


\section{Ex-Vivo Porcine Experiments}

The experiments on porcine eyes were prepared as follows. Enucleated porcine eyes were purchased from a local abattoir the morning of experiments. The eyes were stored on ice before being used. The eyes were fixed on styrofoam using pin needles. A pars plana vitrectomy was performed in all cases. A $20 \mathrm{G}$ trocar for infusion, 25G for chandelier illumination, and $20 \mathrm{G}$ for the access into the posterior space were inserted $3 \mathrm{~mm}$ from the limbus at 10,2 and 4 o'clock respectively. Triamcinolone acetonide (Sigma Aldrich, St. Louis, MI) was injected into the vitreous cavity using a $25 \mathrm{G}$ needle, to help visualize the vitreous humor. When the opacity of the lens deteriorated during experiments, the eye was discarded and a new eye was prepared. After vitrectomy, the porcine eyes were placed in the center of the workspace of the OctoMag, and the MIU and cannula were advanced into the $20 \mathrm{G}$ access trocar, before being fixed by locking the MIU articulated arm. The cannula was advanced until it was visible on the fundus image, before enabling magnetic navigation. The cornea was regularly irrigated with phosphate buffer solution (PBS) during transcorneal OCT visualization.

\section{RESUlts}

\section{A. Theoretical Achievable Workspace}

We used the kinematic model described in III-A to simulate the reachable workspace of the cannula. In the following, the vectors are in the coordinate frame of the camera with the $z$-axis corresponding to the optical axis of the camera, or the dorsal-ventral axis of the eye. The $x$-axis corresponds to the temporal-nasal and the $y$-axis to the superior-inferior axes of the eye. The maximum field intensity was set to $40 \mathrm{mT}$. In the workspace plots of Fig. 4, the insertion length $L$ is varied from 0 to $L_{\max }=26 \mathrm{~mm}$, while applying a magnetic field. The line traced by the distal position of the cannula is shown.

To represent semi-automated control, we rotated a 3D magnetic field with magnitude $40 \mathrm{mT}$ between -170 and $170^{\circ}$ from the proximal axis, and displayed the achievable distal positions, first in the sagittal plane, followed by the frontal plane. This represents the total achievable workspace that can be obtained for a maximum field of $40 \mathrm{mT}$ and can be seen in the left column of Fig. 4.

When using semi-automated control, the workspace is more limited because the field is forced to point inside the image plane, in order to handle the multiple possible magnetic fields which achieve the same projected distal image point. To simulate the workspace in such a situation, we swept a 1D magnetic field component $\eta$ between $-40 \mathrm{mT}$ and $40 \mathrm{mT}$, while applying a fixed downwards field $b_{z}=-15 \mathrm{mT}$ along the dorsal-ventral axis as shown in (22). The field was scaled such that it never exceeded the maximum allowed field as shown in (18).

$$
\mathbf{b}^{\prime}=\left[\begin{array}{lll}
\eta & 0 & b_{z}
\end{array}\right]^{T}
$$

The workspace is shown in the right column of Fig. 4.

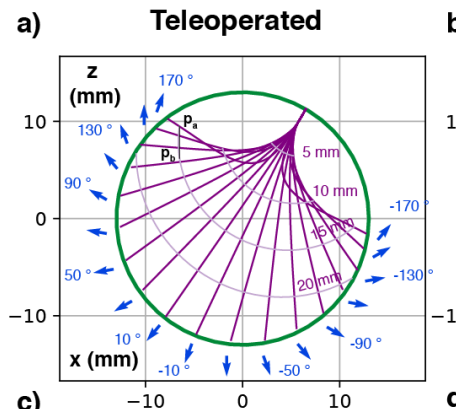

b) Semi-automated
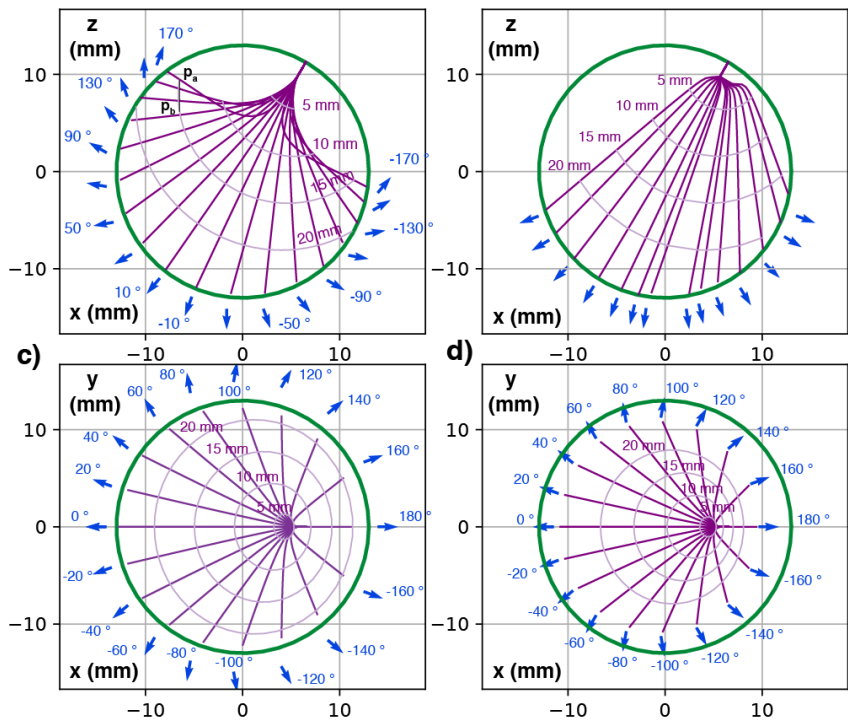

Fig. 4. Illustration of maximum achievable workspace. The dark purple lines show the cannula distal position with increasing length for a given magnetic field. In light purple, the isolines of equal length. The green circles show a diameter of $2.6 \mathrm{~cm}$ corresponding to the average human eyeball diameter. The blue arrows show the magnetic field direction. Top $(\mathrm{a}, \mathrm{b})$, the magnetic field is manipulated in the sagittal plane. Bottom (c,d), the magnetic field is manipulated in the frontal plane. Left $(a, c)$, teleoperated control: the field can be rotated in any direction. Right (b,d), semi-automated control: the field is constrained to always point downwards.

\section{B. Distal Position Precision}

We determined the minimum precision that could be obtained with purely magnetic navigation. The cannula was placed in an open-space and observed with a calibrated microscope. The length of the cannula was set to $24 \mathrm{~mm}$, corresponding to an average diameter of the human eye. We applied a magnetic field of intensity $40 \mathrm{mT}$ along the proximal axis, to emulate worst-case conditions, and tracked the position of the end effector using image processing. There was a positional jitter of maximum amplitude $6 \mu \mathrm{m}$, which could be attributed to the current noise of the eMNS amplifier. The precision of the linear positioner is $1 \mu \mathrm{m}$ according to the manufacturer.

\section{Safety of the Microcannula}

Due to the flexibility of the elastic microcannula, the microcannula should exert much smaller forces in the retina in the worst case, when compared to conventional rigid vitreoretinal instruments. We estimated that these forces should not exceed $2.94 \mathrm{mN}$, as detailed in the appendix. In contrast, the forces applied during conventional retinal surgery are higher with [32] reporting values on the order of $56 \mathrm{mN}$ and $357 \mathrm{mN}$ in the tangential and normal directions respectively, for manual retinal membrane peeling. Our values are upper bounds on the possible forces that can be delivered to the retina, while the forces that can be delivered by existing tools, including those manipulated by RCM robots are much higher due to tool rigidity. 
In order to further demonstrate this claim, the following experiment was performed. The cannula was inserted into the posterior eye and was advanced until the distal capillary was in contact with the retinal tissue. The cannula was further advanced so that buckling occurred on the flexible shaft. The magnetic field was rotated rapidly, causing the cannula to slide, and impact the retina at several points. No retinal tears or holes could be observed during this experiment. In comparison, scraping the same retina with a rigid vitrectromy cutter caused a noticeable retinal tear. A video of the experiment can be seen in supplementary video 5 .

\section{Semi-automated Navigation of the Microcannula}

Semi-automated control was demonstrated in a dry eye phantom (Gulden Ophthalmics, Elkins Park, PA) containing an artificial lens simulating the crystalline lens of the eye. Holes were drilled through the simulated sclera of the phantom into which $25 \mathrm{G}$ and $20 \mathrm{G}$ vitrectomy trocars were inserted. Illumination of the cavity was performed with a $25 \mathrm{G}$ chandelier light (Alcon, Forth Worth, TX). The two-camera system was mounted on the camera port of the microscope. Visualization through the pupil was performed with a wide-angle viewing system. The cannula was introduced through a $20 \mathrm{G}$ trocar into the cavity of the phantom. The microscope and wideangle viewing system were adjusted to obtain a clear view of the fundus. After adjusting the optics, we performed the pre-experiment calibration explained in IV-C.

A target location was selected on the fundus image by moving a handheld joystick controller. Automatic control was toggled on, causing the cannula to align in the direction of the target point. By manually inserting the cannula via the MIU, the catheter was advanced towards the target position. The automatic magnetic navigation of the end-effector allowed the operator to focus solely on inserting the cannula, until it came into contact with the simulated retinal surface. Contact was assessed by observing visual cues such as shadows cast on the retinal surface, or slight buckling of the flexible tool. We demonstrated semi-automated navigation to six different points on the retinal surface. Quantitative assessment of the positioning accuracy is impossible, since the task is shared by both the operator and the controller, and since the feedback control is highly dependent on the tracking accuracy, which is itself dependent on the particular environment found inside the eye cavity. Nevertheless, the qualitative performance is satisfactory as can be seen in footage of the semi-automated experiment, included in supplementary video 2 .

\section{E. Microscope-guided Subretinal Injections}

We demonstrated the ability to safely perform SRI with the microcannula in experiments on enucleated porcine eyes. The preparation of the porcine eyes for experimentation is described in IV-D. The cannula was attached to a $1 \mathrm{~mL}$ syringe and was primed with PBS. After performing a core-vitrectomy, the cannula was inserted with the MIU through a $20 \mathrm{G}$ vitrectomy trocar. The magnetic field was adjusted using the teloperation mode so that the microcannula pointed towards the center of the retinal visual streak (the porcine equivalent

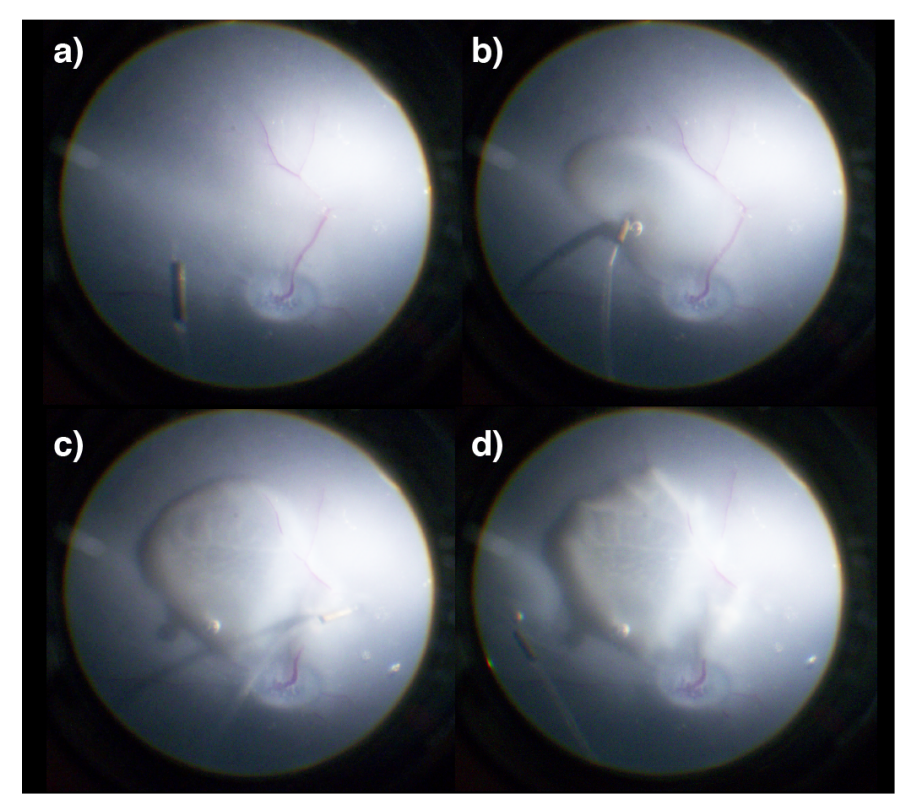

Fig. 5. Microscope-guided subretinal injections. a) The cannula was inserted into the eye via the trans-conjunctival trocar. b) The cannula was navigated to the central visual streak and the first subretinal bleb was created. c) A second subretinal bleb was created on the temporal side of the superior arcade. d) A third bleb was created on the nasal side.

of the macula), on the nasal side of the superior retinal arcade. The cannula was advanced using the MIU towards the retina, with coarse $500 \mu \mathrm{m}$ steps followed by $100 \mu \mathrm{m}$ steps once close to the retina. The cannula was advanced until it was pressing against the retinal surface. An injection volume of $50 \mu \mathrm{L}$ was delivered manually via the syringe, which formed a visible subretinal bleb. The same injection volume was used for all following injections. We navigated the cannula to a second location on the temporal side of the arcade and performed a second injection. A secondary bleb was formed, but formed a connection and enlarged the first bleb. The cannula was then navigated to a location on the nasal side of the visual streak where a third injection was performed, forming a separate bleb. While particular care was made to prime the cannula prior to injection, two small air-bubbles were formed during the experiment. The steps of the injection experiment are shown in Fig. 5. A video of the experiment can be seen in supplementary video 3 .

\section{F. OCT-guided Subretinal Injections}

Experiments were also performed under OCT visualization. The eyes were prepared identically as for the microscopeguided experiments. The microscope was replaced with a spectral-domain OCT (Spectralis OCT, Heidelberg Engineering, Heidelberg, Germany). The cannula was introduced into the eye and manipulated until it was visible in the fundus image of the OCT. It was then inclined towards the retina using the teleoperation mode and advanced towards the retina using steps of $100 \mu \mathrm{m}$ on the MIU. Once the cannula came into contact with the retina, a small indentation could be visible at the site of the retinomy (see Fig. 6 a)). The cannula was further inserted through the retinomy in steps of $10 \mu \mathrm{m}$ using 


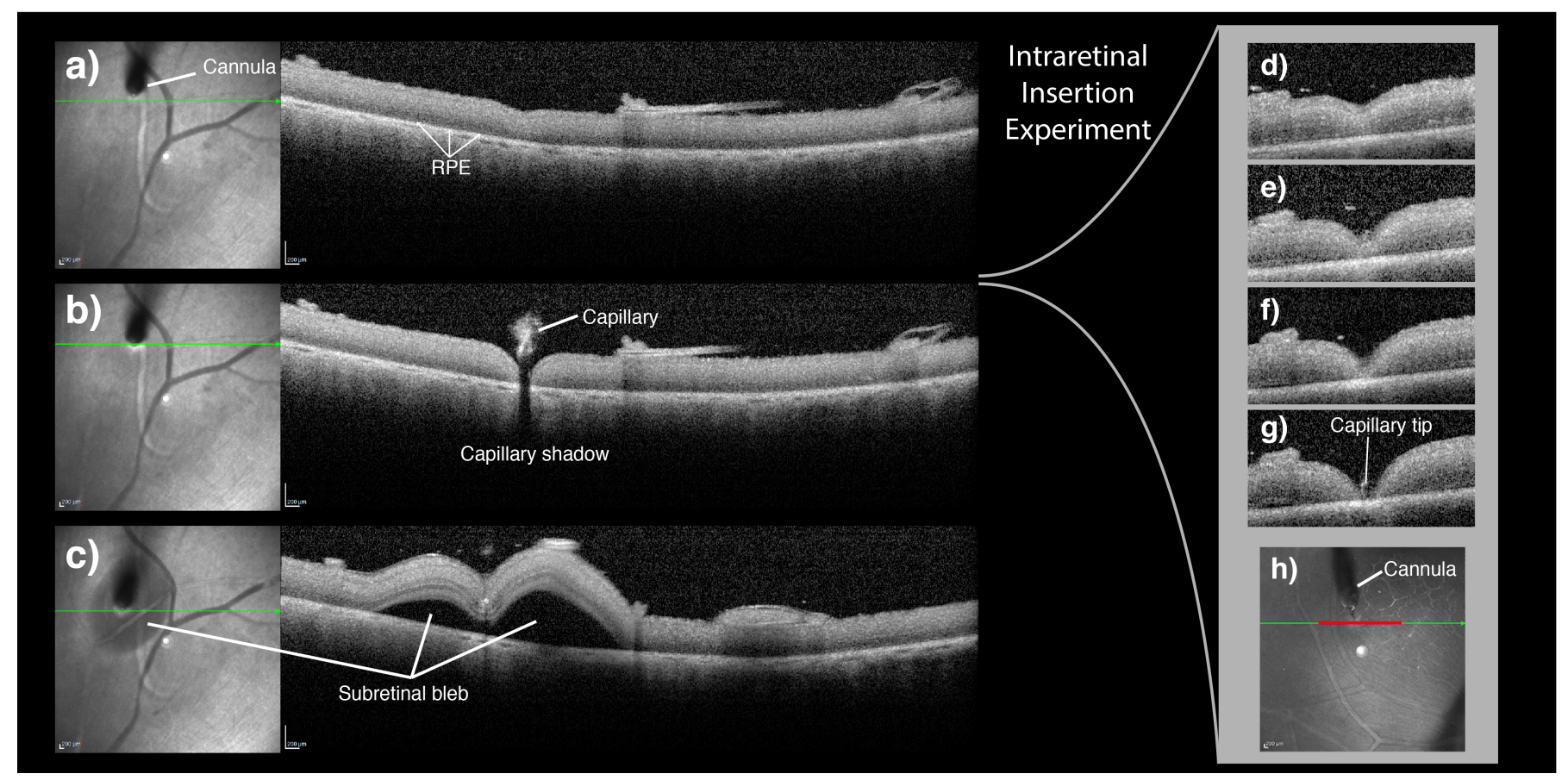

Fig. 6. OCT-guided experiments. a) - c) Injection in the porcine retina. On the left, the fundus image with a green line showing the OCT B-scan line. On the right, the corresponding OCT B-scan. a) The cannula is advanced to slightly indent the retina. b) The cannula is advanced to penetrate the neuro-sensory retina. c) An injection is performed and the subretinal bleb is visible. d) - g) OCT B-scans showing the cannula slowly being advanced towards the RPE using the MIU. h) Fundus image corresponding to d) - g). The red line shows the B-scan section.

the MIU until the indentations reached the RPE. Finally, an injection of $50 \mu \mathrm{L}$ of PBS was delivered, causing the neurosensory retina to lift from the RPE and a subretinal bleb to form (see Fig. 6 c)). The pressure of the cannula against the retina caused a "dimple" to form in the middle of the bleb which was relieved once the cannula was retracted.

In order to show ability to precisely control the insertion of the cannula through the neural layers of the retina, the cannula was driven to the center of the fundus image of the OCT. We then made steps of $10 \mu \mathrm{m}$ with the MIU. The images of Fig. 6 d) - g) were taken after 14, 26, 33, and 37 steps respectively. One can clearly see that the neural layers of the retina are indented as the cannula traverses the retina. The tip of the distal capillary is also visible in some OCT Bscans, allowing the operator to precisely place the cannula at a desired depth in the retina, an important feature for performing safe SRI. A video of the OCT-guided experiments can be seen in supplementary video 4 .

\section{DISCUSSION}

The control paradigm used in this work falls under the telemanipulation category, essentially by requirement. Nevertheless, we believe telemanipulation shows more promise over comanipulation approaches, since it allows for the design of interfaces that are purpose-built, rather than emulating the experience of handheld tools.

With a lateral precision in the $10 \mu \mathrm{m}$ range, the system has a precision comparable to existing robotic systems for vitreoretinal surgery [33]. Such precision is largely sufficient for SRI tasks, and means that the system could also be used for retinal vein cannulation, a procedure that has been targeted by other robotic systems due to high positional precision requirements [34]. The depth of the cannula could be controlled with micrometer precision using the piezo-actuated MIU. Combined with intraoperative OCT visualization, this allows the cannula to be placed at a desired depth in the retinal layers, a feature that would be impossible to do with manual subretinal cannulas. Correct placement of the cannula is important for ensuring that a subretinal bleb is formed safely in the SRS, and does not cause damage to the RPE underneath. It is also important to ensure that therapeutics are injected into the subretinal space and do not escape into the vitreous cavity or the suprachoroidal space [10].

The microcannula is both highly dexterous and very flexible. It has the same benefits of tremor removal and position-holding features of other proposed robotic systems. In contrast to others, it does not require rotations around a RCM and does not result in any movement of the insertion trocars, which have been shown to cause mechanical trauma at the sclerotomies [19]. Increased scleral forces could potentially increase the risk of retinal breaks, hypotony and inflammation. The enhanced flexibility of the tool also increases safety compared to rigid tools, which can cause retinal tears in the event of unwanted movements.

Automatic control is often proposed in the context of task automation, but in this work it is particularly helpful as a human-robot interaction tool. Besides, the MIU is always human-operated in a shared-control strategy. Our experience has shown that navigation of the cannula using 3D rotations of the magnetic field can be cumbersome, since the operator needs to reason in three dimensions even though only two are 
visible, and due to the singularities inherent to 3D rotation parametrizations. Semi-automated control should serve to reduce cognitive burden, since the operator need only manipulate a 2D target point on the image, which can be done with a regular joystick or gamepad interface. Evaluation of the usefulness of this feature could be performed in future multiuser studies. The MIU can be controlled with a separate knob or set of buttons, making the control of the cannula a userfriendly experience, even with a single hand. In cases where semi-automatic control does not yield satisfactory results, such as when navigating to points outside its range of operation shown in Fig. 4, one can always revert to the teleoperation mode.

Due to the high flexibility of the cannula, it achieves a workspace covering a significant portion of the eye. Estimates from the workspace analysis of $\mathrm{V}$-A showed that the cannula could bend upwards of $90^{\circ}$ from the proximal tangent vector and cover the entire posterior hemisphere of the eye. When using semi-automated control, the workspace is more limited since the cannula is constrained to point downwards, but areas visible in wide-angle fundus image should largely be reachable from a single sclerotomy. It may be desireable however to introduce the tool through different sclerotomies, to ease access to certain regions. For example, to avoid having to the bend cannula backwards, which presents some kinematic instabilities [22, p. 20], one may prefer to use a second sclerotomy on the opposite side. To visualize the most peripheral areas of the retina, techniques such as scleral indentation or endoscopic visualization may also be helpful.

Magnetic navigation has the added benefit that it requires no moving parts beyond the linear motion of the MIU. Reaching high dexterity with a RCM constrained robot can result in large motions of the manipulator around the patient. This may have safety and patient acceptance considerations, as patients who do not undergo full anaesthesia may be uncomfortable with rapid mechanical motions of a robot close to their facial area. Magnetic navigation does have its disadvantages. Precautions would have to be made such as avoiding the use of tools made from magnetic materials while the eMNS is in use. This work has been conducted in the OctoMag, an eMNS designed for ex-vivo experimental work. A larger eMNS would be required for navigation in a human patient. Such clinicalscale systems are more complex and expensive since the electromagnets are much larger and required active cooling, but systems have already been commercialized for applications in cardiology [22, p. 11]. Using a large-scale eMNS should not lead to any changes in performance, given that it can achieve equivalent magnetic fields of $40 \mathrm{mT}$. Nevertheless there may be unforeseen challenges integrating an eMNS in an ophthalmic operating theater with regards to compatibility with existing equipment and procedures. There is also fixed cost associated with installing the eMNS, which would have to be absorbed by hospitals in order to perform magneticallynavigated procedures.

\section{CONCLUSION}

Subretinal injections are a promising surgical technique for delivering the next generation of therapeutics for treating retinal disorders such as AMD. They represent a difficult procedure with hefty risks, and new technological tools such as robotic manipulation and intraoperative OCT have been proposed to abate such challenges. We developed a remotely navigated magnetic microcannula for performing subretinal injections. We showed in dry eye phantoms and ex-vivo porcine eyes that the cannula can be navigated precisely, safely, and intuitively over a large portion of the posterior eye using microscope and intraoperative OCT visualization.

Future work will be directed at adapting the system to clinical settings for potential operations on human patients. Attention will be focused on integrating the system with new eMNS designs that are compatible with operating theaters, while increasing the user-friendliness of the system to medical personnel.

\section{APPENDIX}

Theoretical Analysis of the Forces ApPliEd on THE RETINA

Consider a bent microcannula of length $L_{f}=21 \mathrm{~mm}$ that is in contact with the retina surface, such it is clamped at both the proximal end at $\mathbf{O}_{p}$ and at the point of contact at the distal end. The scenario is shown in Fig. 7. The angle between the distal tangent vector and $\mathbf{t}_{0}$ is $\varphi_{0}$. In the following, we take an example where the cannula is bent such that $\varphi_{0}=45^{\circ}$. The total set of forces acting at the cannula tip are

- $F_{m}$ : the force associated with a magnetic torque $\tau_{m}$ acting on the distal magnet's magnetic moment $M$.

- $F_{e}$ : The force associated with the deflection of the flexible part of the cannula acting on the retina surface. This force is zero when the cannula is straight.

- $F_{r}=F_{m}+F_{e}$ : The resulting sum of forces acting on the retina.

- $F_{r}^{\|}$and $F_{r}^{\perp}$ : The tangential and normal components of $F_{r}$.

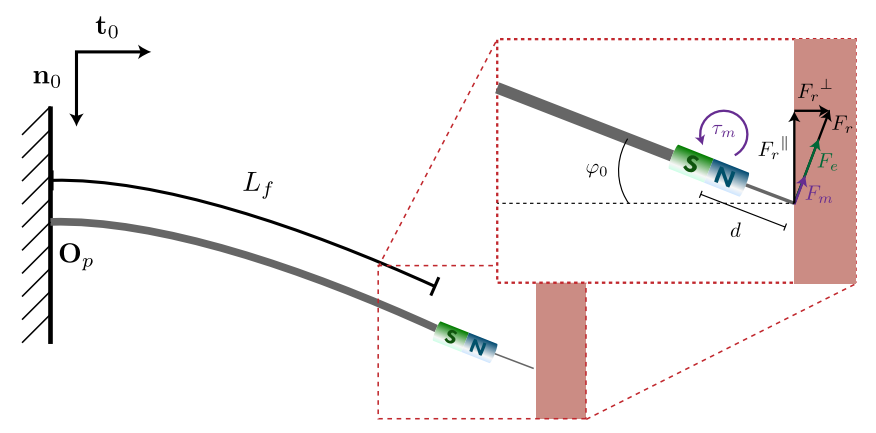

Fig. 7. Diagram showing the forces applied on the retina

In the following calculations, it is assumed that $F_{r}$ can be split independently into the effect of the cannula deflection $F_{e}$, and the effect of the magnetic torque $F_{m}$. In reality, these forces are coupled, and the resulting force $F_{r}$ should be lower than the one calculated below. For simplicity we do not consider the coupling, since we are concerned with estimates in the worse-case. We make the following assumptions

1) The cannula bends in the plane spanned by $\mathbf{t}_{0}$ and $\mathbf{n}_{0}$. 
2) Forces and torques acting on the cannula do not cause it to move.

3) The cannula is clamped at its proximal end at $\mathbf{O}_{p}$.

4) The magnetic torque acts on the magnet's center of gravity.

5) The distance between the magnet center of gravity and the distal tip of the cannula, in contact with the retina is $d$.

6) The retina is flat and rigid. The contact forces between the retina and the distal point of the cannula are perpendicular to the distal tangent of the cannula.

The relationship between the length of the flexible segment $L_{f}$ and the angle $\varphi(s)$ between a point on the cannula $\mathbf{r}(s)$ and $\mathbf{t}_{0}$ at arclength $s$ is defined by the following integral [35].

$$
L_{f}=\sqrt{\frac{E_{f} I_{f}}{2 F_{e}}} \int_{0}^{\varphi_{0}} \frac{d \varphi}{\sqrt{\sin \varphi_{0}-\sin \varphi}}
$$

where $E_{f}$ is the Young's modulus and $I_{f}$ is the second moment of area of the flexible segment. $F_{e}$ can be calculated using incomplete Jacobi elliptic integrals of the first kind. Using the parameters specified above yields $F_{e}=0.108 \mathrm{mN}$.

The following estimates the worst case forces that are associated with the magnetic torques acting on the distal magnet. Using the same magnetic moment $M=0.15 \mathrm{~A} \mathrm{~m}^{2}$ as in the paper, consider a magnetic field of magnitude $B_{0}=40 \mathrm{mT}$ orthogonal to the magnetic moment of the magnet such that the magnetic torque is maximum. The force resulting from $\tau_{m}$ is $F_{m}=\frac{M B_{0}}{d}$ yielding $F_{m}=2.83 \mathrm{mN}$. The total resulting force is then $F_{r}=2.94 \mathrm{mN}$ with tangential and normal forces of $2.08 \mathrm{mN}$ respectively.

\section{ACKNOWLEDGMENT}

We would like to thank Katrin Voelter and Simon Pot from Tierspital Zurich for their consultation throughout the project.

This work was partially supported by the Swiss National Science Foundation through grant number 200021_165564, and the ERC Advanced Grant 743217 Soft Micro Robotics (SOMBOT).

\section{REFERENCES}

[1] S. R. Flaxman et al., "Global causes of blindness and distance vision impairment 19902020: a systematic review and meta-analysis," The Lancet Global Health, vol. 5, no. 12, pp. e1221-e1234, 2017.

[2] W. L. Wong et al., "Global prevalence of age-related macular degeneration and disease burden projection for 2020 and 2040: A systematic review and meta-analysis," The Lancet Global Health, vol. 2, no. 2, pp. e106-e116, 2014.

[3] S. S. Thakur et al., "Intravitreal drug delivery in retinal disease: are we out of our depth?" Expert opinion on drug delivery, vol. 11, no. 10, pp. 1575-90, 2014.

[4] D. F. Kiernan and W. F. Mieler, "The use of intraocular corticosteroids," Expert Opinion on Pharmacotherapy, vol. 10, no. 15, pp. 2511-2525, 102009.

[5] B. Roska and J. A. Sahel, "Restoring vision," Nature, vol. 557, no. 7705, pp. 359-367, 2018.

[6] T. Ong et al., "Adeno-Associated Viral Gene Therapy for Inherited Retinal Disease," p. 34, 2019.

[7] Clinicaltrials.gov, "RGX-314 Gene Therapy for Neovascular AMD Trial," 2019.

[8] L. Da Cruz et al., "Phase 1 clinical study of an embryonic stem cell-derived retinal pigment epithelium patch in age-related macular degeneration," Nature Biotechnology, vol. 36, no. 4, pp. 1-10, 2018.
[9] M. S. Humayun, E. de Juan, and G. Dagnelie, "The Bionic Eye: A Quarter Century of Retinal Prosthesis Research and Development," Ophthalmology, vol. 123, no. 10, pp. S89-S97, 102016.

[10] K. Xue et al., "Technique of retinal gene therapy: Delivery of viral vector into the subretinal space," Eye (Basingstoke), vol. 31, no. 9, pp. 1308-1316, 2017.

[11] A. Chan et al., "Normal macular thickness measurements in healthy eyes using stratus optical coherence tomography," Archives of Ophthalmology, vol. 124, no. 2, pp. 193-198, 2006.

[12] S. P. Singhy and C. N. Riviere, "Physiological tremor amplitude during retinal microsurgery," Proc. IEEE Annual Northeast Bioeng. Conf., pp. 171-172, 2002.

[13] J. L. Davis et al., "Surgical Technique for Subretinal Gene Therapy in Humans with Inherited Retinal Degeneration," Retina, p. 1, 2019.

[14] R. Tadayoni, "Intraoperative OCT: Would You Like Some Extra Information?" Ophthalmology Retina, vol. 2, no. 4, pp. 261-262, 2018.

[15] M. D. Fischer et al., "Evaluation of an Optimized Injection System for Retinal Gene Therapy in Human Patients," Human Gene Therapy Methods, vol. 27, no. 4, pp. 150-158, 2016.

[16] M. A. Nasseri, M. Maier, and C. P. Lohmann, "A targeted drug delivery platform for assisting retinal surgeons for treating Age-related Macular Degeneration (AMD)," Conf. Proc. 39th IEEE Eng. Med. Biol. Soc., pp. 4333-4338, 2017.

[17] J. Smits et al., "Robot-Assisted Subretinal Surgery: initial in-vivo animal validation," pp. 91-92, 2019.

[18] T. L. Edwards et al., "First-in-human study of the safety and viability of intraocular robotic surgery," Nature Biomed. Engineering, vol. 2, no. 9, pp. 649-656, 2018.

[19] C. He et al., "User Behavior Evaluation in Robot-Assisted Retinal Surgery," 27th IEEE Int. Symp. Rob. Human Interactive Commun., pp. 174-179, 2018.

[20] G. Bradski, "OpenCV Library," Dr. Dobb's Journal of Software Tools, 1999.

[21] M. P. Kummer et al., "Octomag: An electromagnetic system for 5-DOF wireless micromanipulation," IEEE Trans. Robot., vol. 26, no. 6, pp. 1006-1017, 122010.

[22] C. Chautems, "Magnetic Catheters for Cardiac Arrhythmia Ablations," Ph.D. dissertation, ETH Zurich, 2019.

[23] I. Tunay, "Modeling magnetic catheters in external fields," in Annual International Conference of the IEEE Engineering in Medicine and Biology - Proceedings, vol. 26 III. IEEE, 2004, pp. 2006-2009.

[24] A. J. Petruska, J. Edelmann, and B. J. Nelson, "Model-Based Calibration for Magnetic Manipulation," IEEE Trans. on Magnetics, vol. 53, no. 7, pp. 1-1, 72017.

[25] S. L. Charreyron, B. Zeydan, and B. J. Nelson, "Shared Control of a Magnetic Microcatheter for Vitreoretinal Targeted Drug Delivery," in Internal Conference on Robotics and Automation, Singapore, 2017, pp. 4843-4848.

[26] I. Tunay, "Position control of catheters using magnetic fields," Proc. IEEE Int. Conf. on Mechatronics, pp. 392-397, 2004.

[27] S. L. Charreyron et al., "A Magnetically Steered Endolaser Probe for Automated Panretinal Photocoagulation," Robotics and Automation Letters, vol. 4, no. 2, pp. 284-290, 2018.

[28] R. Hartley and A. Zisserman, Mulitple View Geometry, 2nd ed. Cambridge University Press, 2003.

[29] I. Bekerman, P. Gottlieb, and M. Vaiman, "Variations in Eyeball Diameters of the Healthy Adults," Journal of Ophthalmology, vol. 2014, 2014.

[30] I. Sanchez et al., "The parameters of the porcine eyeball," Graefe's Archive for Clinical and Experimental Ophthalmology, vol. 249, no. 4, pp. 475-482, 2011.

[31] V. Madanagopalan et al., "Influence of orientation of the external linear incision created by the 25 -gauge trocar and related factors on sclerotomy closure: A clinical and optical coherence tomographic study," Indian journal of ophthalmology, vol. 66, no. 12, pp. 1809-1814, 2018.

[32] A. S. Jagtap and C. N. Riviere, "Applied force during vitreoretinal microsurgery with handheld instruments." Conf. Proc. IEEE Eng. Med. Biol. Soc., vol. 4, no. 1, pp. 2771-2773, 2004.

[33] M. D. de Smet et al., "Human/robotic interaction: vision limits performance in simulated vitreoretinal surgery," Acta Ophthalmologica, pp. $1-7,2018$.

[34] M. D. De Smet et al., "Robotic assisted cannulation of occluded retinal veins," PLOS ONE, vol. 11, no. 9, pp. 1-16, 2016.

[35] T. Beí Endez, C. Neipp, and A. Beí Endez, "Large and small deflections of a cantilever beam," Tech. Rep., 2002. 